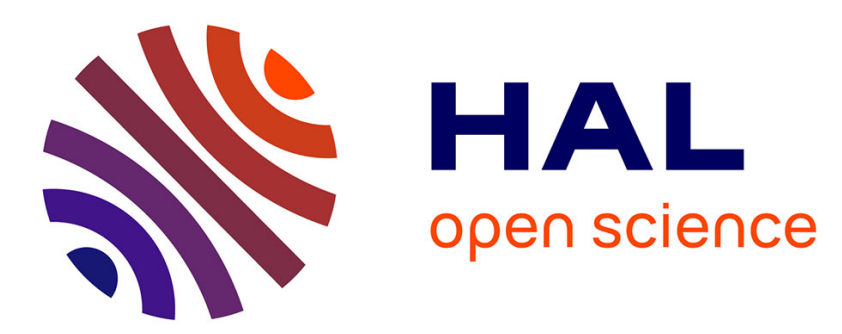

\title{
On the forced response of multi-layered systems using the modified wave finite element method
}

J.-M Mencik

\section{To cite this version:}

J.-M Mencik. On the forced response of multi-layered systems using the modified wave finite element method. The Ninth International Conference on Computational Structures Technology, Sep 2008, Athens, Greece. 10.4203/ccp.88.46 . hal-01202829

\section{HAL Id: hal-01202829 \\ https://hal.science/hal-01202829}

Submitted on 21 Sep 2015

HAL is a multi-disciplinary open access archive for the deposit and dissemination of scientific research documents, whether they are published or not. The documents may come from teaching and research institutions in France or abroad, or from public or private research centers.
L'archive ouverte pluridisciplinaire HAL, est destinée au dépôt et à la diffusion de documents scientifiques de niveau recherche, publiés ou non, émanant des établissements d'enseignement et de recherche français ou étrangers, des laboratoires publics ou privés. 


\title{
On the forced response of multi-layered systems using the modified wave finite element method J.-M. Mencik
}

\author{
ENI Val de Loire, Université François Rabelais de Tours, LMR, Blois, France
}

\begin{abstract}
In this paper, the forced response of multi-layered elastic waveguides is addressed. The formalism uses global wave modes as a projection basis. These global modes are numerically constructed from the local wave modes of the layers within the framework of the modified wave finite element (MWFE) method. The method uses a dynamic substructuring scheme which allows the dynamics of each layer cross-section to be projected onto a reduced local wave mode basis with appropriate dimension. The MWFE method is used to predict the forced response of multi-layered systems. The convergence of the model with regard to the size of the wave mode basis is discussed. Numerical simulations and comparisons with standard techniques show the pertinence of the model.
\end{abstract}

Keywords: multi-layered systems; forced response; wave finite elements; dynamic substructuring; global wave modes; local wave modes.

\section{Introduction}

Multi-layered systems are extensively used in many engineering areas (automotive and aeronautic fields among others). Such systems constitute interesting light and stiff solutions for mechanical product design. Understanding and predicting their vibratory behavior has raised much interest in the literature. The Finite Element Method (FEM) [1,2] is the most employed tool to deal with the dynamic behavior of such structures. Nevertheless, excessive computational cost associated with large size model resolution, involved in time response calculation for instance, constitutes one major limitation of the method; another limitation of the FEM is related to model reduction aspects where the convergence of the standard reduced modal basis is not necessarily satisfied, a priori, in the short wavelength domain. As an alternative, the dynamic analysis of multi-layered systems based on wave mode descriptions seems interesting since it requires the treatment of relatively small models whose sizes are related to cross-section dynamics only. The technique appears particularly well suited 
for the short wavelength domain as the dynamics of systems can be accurately described by highly convergent reduced bases containing the essential wave motions [3].

A numerical technique for wave mode description in slender systems with simple or complex cross-section has been proposed by Mead [4,5] and Zhong \& Williams [6]. The technique is based on a classic 3D finite element scheme of a typical cell and provides wave properties solving either a quadratic or linear eigenvalue problem. The formulation, termed Wave Finite Element (WFE), has been applied to elastic systems [7, 8], pipes with internal fluid [3] and laminates [9]. The WFE formulation appears optimal to describe the dynamic behavior of homogeneous systems. This is explained because the structural/acoustic fields can be projected onto specific reduced bases which contain non-dependent eigenvectors and whose dimension correlates the global vibration scale of the cross-section. On the other hand, the formulation can suffer from numerical issues when multi-layered systems are dealt with, because it provides wave bases whose dimension overestimates the dynamics of the global crosssection. This is especially verified when the layers exhibit multiscale behavior. In this case, the wave mode bases contain dependent vectors - namely the physical motions and a set of spurious modes - which generate singular problems. To solve the WFE difficulties, a dynamic substructuring technique, allowing the dynamics of each layer cross-section to be projected on a local wave mode basis with appropriate dimension, has been proposed in ref. [10]. The formulation, termed Modified Wave Finite Element (MWFE), allows the global wave modes of a given multi-layered system to be constructed from the local wave mode bases attached to the uncoupled layers.

The use of the MWFE formulation to predict the forced response of multi-layered systems is the main object of this paper. In Section 2, we recall the classic wave finite element (WFE) technique and discuss on the numerical issue of the method. The MWFE formalism, as derived in ref. [10], is presented in Section 3. In Section 4, we apply the MWFE formalism to describe the forced response of systems. The Neumann to Dirichlet problem associated with the formulation is established. Numerical simulations and comparisons with standard FE techniques are finally presented in Section 5 .

\section{WFE formulation}

\subsection{Statement of the elastic problem}

The study is based on a bounded elastic and dissipative slender structure, as illustrated in Figure 1, composed of an arbitrary number (say $M$ ) of connected straight homogeneous layers with constant cross-sections. The global heterogeneous structure is assumed to vibrate linearly, under harmonic excitation at the frequency $\omega / 2 \pi$, around a static equilibrium taken as the reference configuration. 

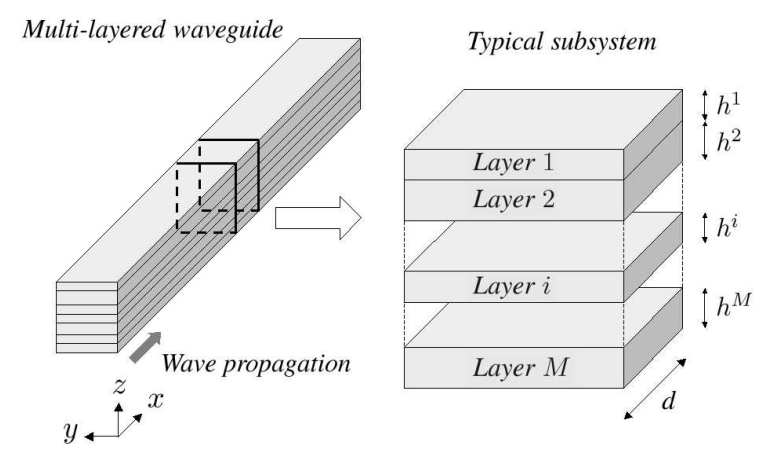

Figure 1: Illustration of a multi-layered elastic system with a rectangular cross-section.

A typical subsystem of length $d$ extracted from the global multi-layered system is used (see Figure 1). The finite element model of this local subsystem leads to the following standard dynamic equilibrium equation:

$$
\mathrm{Dq}=\mathrm{F},
$$

where $\mathbf{D}$ denotes the dynamic stiffness operator of the discretized subsystem; $\mathbf{q}$ and $\mathrm{F}$ are the displacements and the forces defined at the subsystem degrees of freedom (DOFs). Here, the left and right boundaries of the subsystem are assumed to contain the same nodal distribution: the number of DOFs for each boundary is denoted $n$. Since internal nodes, i.e. which do not belong to the left and right boundaries, are not excited, the dynamic equilibrium (1) can be condensed to give [11]:

$$
\mathbf{u}_{\mathrm{R}}=\mathrm{Su}_{\mathrm{L}}
$$

where $\mathbf{u}_{\mathrm{L}}=\left(\left(\mathbf{q}_{\mathrm{L}}\right)^{T}\left(-\mathbf{F}_{\mathrm{L}}\right)^{T}\right)^{T}$ and $\mathbf{u}_{\mathrm{R}}=\left(\left(\mathbf{q}_{\mathrm{R}}\right)^{T}\left(\mathbf{F}_{\mathrm{R}}\right)^{T}\right)^{T}$ are $(2 n \times 1)$ state vectors and subscripts $\mathrm{L}$ and $\mathrm{R}$ refer to the left and right boundaries, respectively. $\mathbf{S}$ is a $(2 n \times 2 n)$ symplectic matrix given by

$$
\mathbf{S}=\left[\begin{array}{c|c}
-\left(\mathbf{D}_{\mathrm{LR}}^{*}\right)^{-1} \mathbf{D}_{\mathrm{LL}}^{*} & -\left(\mathbf{D}_{\mathrm{LR}}^{*}\right)^{-1} \\
\hline \mathbf{D}_{\mathrm{RL}}^{*}-\mathbf{D}_{\mathrm{RR}}^{*}\left(\mathbf{D}_{\mathrm{LR}}^{*}\right)^{-1} \mathbf{D}_{\mathrm{LL}}^{*} & -\mathbf{D}_{\mathrm{RR}}^{*}\left(\mathbf{D}_{\mathrm{LR}}^{*}\right)^{-1}
\end{array}\right]
$$

where $\mathbf{D}^{*}$ denotes the condensed dynamic stiffness operator. The eigenvectors and eigenvalues of $\mathbf{S}$, namely $\left\{\boldsymbol{\Phi}_{j}\right\}_{j}$ and $\left\{\mu_{j}\right\}_{j}$, are solutions to the following problem:

$$
\mathbf{S} \boldsymbol{\Phi}_{j}=\mu_{j} \boldsymbol{\Phi}_{j} \quad, \quad\left|\mathbf{S}-\mu_{j} \mathbf{I}\right|=\mathbf{0} .
$$

The WFE technique is based on the direct estimation of parameters $\left\{\left(\mu_{j}, \boldsymbol{\Phi}_{j}\right)\right\}_{j}$ obtained by solving (4). The WFE formulation is recalled hereafter.

\subsection{Wave mode description}

We assume that the global system is composed of $N$ identical connected subsystems along axis $x$ (see Figure 1). As discussed above, we also assume the mesh compatibility between subsystems at coupling interfaces. In this sense, the coupling conditions 
between two consecutive subsystems $k$ and $k-1$ write [11]

$$
\mathbf{u}_{\mathrm{L}}^{(k)}=\mathbf{u}_{\mathrm{R}}^{(k-1)} \quad k=2, \ldots, N
$$

which, according to Eq. (2), leads to :

$$
\mathbf{u}_{\mathrm{L}}^{(k)}=\mathbf{S u}_{\mathrm{L}}^{(k-1)} \quad k=2, \ldots, N .
$$

The analysis of the dynamic behavior of the global periodic system is based on Bloch's theorem [12], which states that the solutions $\mathbf{u}_{\mathrm{L}}$ are of the form

$$
\mathbf{u}_{\mathrm{L}}^{(k)}=\mu \mathbf{u}_{\mathrm{L}}^{(k-1)} \quad k=2, \ldots, N .
$$

Inserting (7) into (6) leads to the linear eigenvalue problem (4). The parameters $\left\{\left(\mu_{j}, \boldsymbol{\Phi}_{j}\right)\right\}_{j=1, \ldots, 2 n}$ refer to the wave modes of the global system. The wave mode matrix, namely $\Phi$, can be partitioned as

$$
\Phi=\left[\begin{array}{ll}
\Phi_{\mathrm{q}}^{\text {inc }} & \boldsymbol{\Phi}_{\mathrm{q}}^{\mathrm{ref}} \\
\boldsymbol{\Phi}_{\mathrm{F}}^{\text {inc }} & \boldsymbol{\Phi}_{\mathrm{F}}^{\mathrm{ref}}
\end{array}\right]
$$

where the subscripts $\mathbf{q}$ and $\mathbf{F}$ refer to the displacement and force components, respectively; the superscripts inc and ref refer to the modes which are incident to - and reflected ${ }^{1}$ by - a specific boundary (left or right) of the waveguide, respectively; $\Phi_{\mathbf{q}}^{\text {inc }}$, $\boldsymbol{\Phi}_{\mathbf{F}}^{\text {inc }}, \boldsymbol{\Phi}_{\mathbf{q}}^{\text {ref }}$ and $\boldsymbol{\Phi}_{\mathbf{F}}^{\text {ref }}$ are square $(n \times n)$ matrices.

The wave formulation established above constitutes the framework of the WFE formulation and appears well suited for predicting the spatial distribution of the kinematic variables along the global system. The technique is based on the expansion of either $\mathbf{u}_{\mathrm{L}}^{(k)}$ or $\mathbf{u}_{\mathrm{R}}^{(k)}$ on a reduced wave mode basis $\left\{\widetilde{\boldsymbol{\Phi}}_{j}\right\}_{j=1, \ldots, 2 m}$ extracted from the global wave mode basis $\left\{\boldsymbol{\Phi}_{j}\right\}_{j=1, \ldots, 2 n}(m \leq n)[3]$ :

$$
\mathbf{u}_{\mathrm{L}}^{(k)}=\widetilde{\boldsymbol{\Phi}} \widetilde{\mathbf{Q}}^{(k)} \quad, \quad \mathbf{u}_{\mathrm{R}}^{(k)}=\widetilde{\boldsymbol{\Phi}} \widetilde{\mathbf{Q}}^{(k+1)} \quad k=1, \ldots, N,
$$

where $\widetilde{\mathrm{Q}}$ are the modal amplitudes which can be partitioned into incident and reflected components as $\widetilde{\mathbf{Q}}=\left(\left(\widetilde{\mathbf{Q}}^{\text {inc }}\right)^{T}\left(\widetilde{\mathbf{Q}}^{\text {ref }}\right)^{T}\right)^{T}$. Those components can be obtained via the following governing equation:

$$
\widetilde{\mathbf{Q}}^{\text {inc }(k)}=\widetilde{\boldsymbol{\mu}}^{k-1} \widetilde{\mathbf{Q}}^{\text {inc }(1)} \quad, \quad \widetilde{\mathbf{Q}}^{\mathrm{ref}(k)}=\widetilde{\boldsymbol{\mu}}^{-(k-1)} \widetilde{\mathbf{Q}}^{\mathrm{ref}(1)} \quad k=1, \ldots, N+1
$$

with the boundary conditions

$$
\left.\widetilde{\mathbf{Q}}^{\text {ref }}\right|_{\text {lim }}=\left.\widetilde{\mathbb{C}} \widetilde{\mathbf{Q}}^{\text {inc }}\right|_{\text {lim }}+\widetilde{\mathcal{F}}
$$

Here, $\widetilde{\boldsymbol{\mu}}$ is the diagonal eigenvalue matrix associated with eigenvectors $\left\{\widetilde{\boldsymbol{\Phi}}_{j}\right\}_{j=1, \ldots, m}$; $\widetilde{\mathbb{C}}$ and $\widetilde{\mathcal{F}}$ denote the diffusion matrix and the effects of the excitation sources [3]. In general, to ensure the validity of the expansion (9), the reduced modal basis must contain the modes which are the most solicited by the excitation.

\footnotetext{
${ }^{1}$ This refers to the waves which "appear reflected" by the boundary and which include effective reflected modes, as well as transmitted and excited modes.
} 


\subsection{Discussion}

Due to the boundary value problem (10), the WFE technique consists of projecting the displacements and forces onto wave bases of the form $\left\{\widetilde{\boldsymbol{\Phi}}_{j}\right\}_{j}$ to describe the forced response of systems. The practical implementation of the WFE method consists of meshing a typical cross-section with a sufficient number of nodes to "correctly capture" the cross-section dynamics within a given frequency band. This practical routine was successfully applied to homogeneous systems [11], as each part of a typical crosssection exhibits approximately the same vibratory behavior.

Difficulties arise when multi-layered systems are considered, as a typical crosssection contains parts whose dynamic behavior can greatly differ. This is observed for instance in sandwich beams with a soft core (rubber) surrounded by two stiff skins (steel). In this case, mid-frequency (MF) behavior of the core and low-frequency (LF) behavior of the skins coexist. To apply the WFE formalism, the mesh density of the global cross-section is usually chosen to be sufficiently rich to account for the MF behavior of the core. As a result, the dimension of eigenvalue problem (4) can greatly exceed the sum of the layer dimensions and spurious high order wave modes, whose eigenvalues are extremely large or close to zero, are then generated. This leads to numerical pollution effects (we refer to ref. [10]) and a poor convergence of eigenvalue problem (4) for wave mode extraction.

Those issues can be numerically highlighted by considering the case of a threelayered sandwich beam, with a rectangular cross-section (see Figure 2), consisting of a soft rubber core (layer 2) surrounded by two stiff steel skins (layers 1 and 3). Here, the skins have the same characteristics: height and width, along the $z-$ and $y-$ axes, are $h^{1}=h^{3}=2 \times 10^{-3} \mathrm{~m}$ and $50 \times 10^{-3} \mathrm{~m}$ respectively, while Young's modulus, density and Poisson ratio are $E^{1}=E^{3}=2.1 \times 10^{11} \mathrm{~Pa}, \rho^{1}=\rho^{3}=7850 \mathrm{~kg} / \mathrm{m}^{3}$ and $\nu^{1}=\nu^{3}=0.3$ respectively. The core exhibits the following characteristics: height and width, along the $z-$ and $y-$ axes, are $h^{2}=20 \times 10^{-3} \mathrm{~m}$ and $50 \times 10^{-3} \mathrm{~m}$ respectively, while Young's modulus, density and Poisson ratio are $E^{2}=1.5 \times 10^{6} \mathrm{~Pa}$, $\rho^{2}=950 \mathrm{~kg} / \mathrm{m}^{3}$ and $\nu^{2}=0.3$ respectively. The global system considered through the WFE formulation is assumed to be dissipative - this is modeled by means of a similar loss factor $\eta=0.01$. The wave mode parameters of the system are numerically evaluated with the WFE formulation, considering the finite element model of a typical subsystem extracted from the global multi-layered waveguide. Such a subsystem is shown in Figure 2 and reflects the three coupled layers. The length of the subsystem, along the direction of wave propagation ( $x$-axis), is $d=2 \times 10^{-3} \mathrm{~m}$ and is chosen to be small enough with regard to the wavelengths of the significantly contributing wave modes within the given frequency band [3]. Both layers 1 and 3 (steel skins) of the subsystem are meshed with four linear block elements while layer 2 (soft core) is meshed with sixteen linear block elements. Within the WFE framework, this relatively coarse mesh should be appropriate to yield the classic LF modes of the global system and the first cross-section modes which are associated with the local dynamics of the core cross-section. 


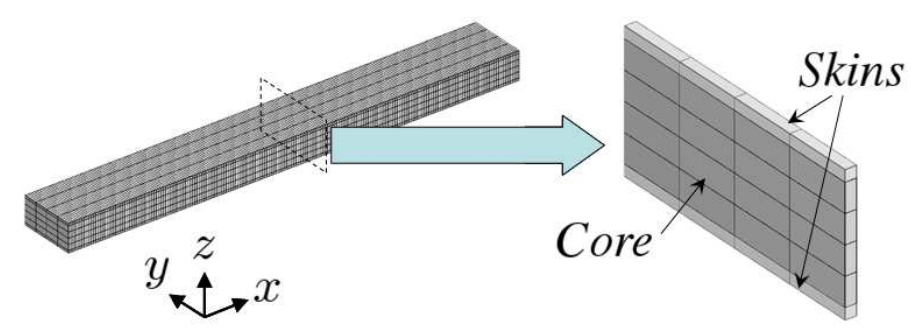

Figure 2: Illustration of a multi-layered elastic system with a rectangular cross-section.

In brief, it can be emphasized that several "quite similar" longitudinal wave modes are generated by the WFE formulation: this is highlighted in Figures 3 and 4 where the classic longitudinal mode and a cross-section mode (i.e. which cuts on at a specific frequency) exhibit approximately the same nature around $600 \mathrm{~Hz}$. Here, the dependency between these two modes is clearly established. Spurious dependent modes are then generated by the WFE formulation. Those modes cause numerical pollution effects and a lack of convergence of the formulation. The issue can be solved when the dynamics of each homogeneous layer is expanded on a local wave mode basis with a suitable dimension. In the present work, this local basis reflects the waves traveling in the layer decoupled from its surroundings and is attached to the finite element model of the associated homogeneous subsystem with free interface. In this sense, the global wave mode basis of the multi-layered system can be constructed using classic substructuring techniques. The method, termed Modified Wave Finite Element (MWFE), is described below.
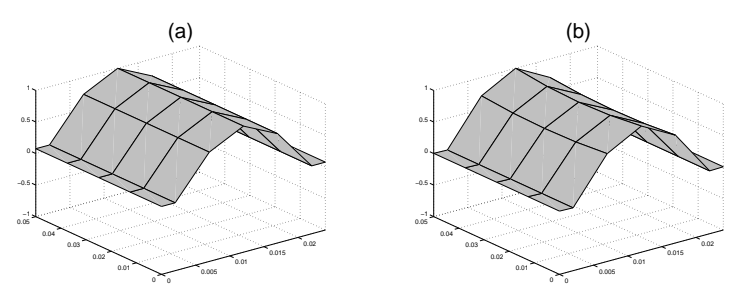

Figure 3: Displacement components (real parts) of the wave mode shapes at $600 \mathrm{~Hz}$ : (a) classic longitudinal mode; (b) cross-section longitudinal mode.
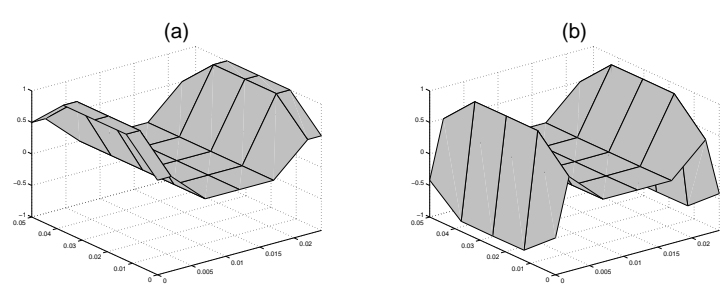

Figure 4: Force components (real parts) of the wave mode shapes at $600 \mathrm{~Hz}$ : (a) classic longitudinal mode; (b) cross-section longitudinal mode. 


\section{Modified Wave Finite Element (MWFE) formulation}

\subsection{Local wave mode description}

We consider a typical multi-layered subsystem $k$ of the global waveguide (see Figure 1). In the present work, the subsystem is assumed to represent a set of $M$ connected straight homogeneous layers, whose left and right boundaries are denoted respectively as $\left\{S_{\mathrm{L}}^{i}\right\}_{i}$ and $\left\{S_{\mathrm{R}}^{i}\right\}_{i}$ : for each layer $i(i=1, \ldots, M)$, the surfaces $S_{\mathrm{L}}^{i}$ and $S_{\mathrm{R}}^{i}$, are assumed to contain the same number of DOFs (see Section 2.1), say $n^{i}$. Let us denote as $\Gamma^{i}$ the coupling interface between each layer $i$ and its surroundings, that is, the set of coupled layers $\{j\}_{j \neq i}$.

The MWFE formulation consists in constructing the global wave mode basis $\left\{\boldsymbol{\Phi}_{j}\right\}_{j}$, attached to the heterogeneous multi-layered subsystem $k$, from a set of reduced local wave mode bases $\left\{\left\{\widetilde{\boldsymbol{\Phi}}_{j}^{i}\right\}_{j=1, \ldots, 2 m^{i}}\right\}_{i}$, having specific dimensions $\left\{2 m^{i}\right\}_{i}\left(m^{i} \leq n^{i} \forall i\right)$ and attached to the set of homogeneous layers $\{i\}_{i}$ with free interfaces $\left\{\Gamma^{i}\right\}_{i}$. For each uncoupled layer $i$, the reduced basis $\left\{\widetilde{\boldsymbol{\Phi}}_{j}^{i}\right\}_{j=1, \ldots, 2 m^{i}}$ is assumed to support the crosssection (either $S_{\mathrm{L}}^{i}$ or $S_{\mathrm{R}}^{i}$ ) dynamics within the given frequency band and is extracted from the full local basis $\left\{\boldsymbol{\Phi}_{j}^{i}\right\}_{j=1, \ldots, n^{i}}$ which is obtained using the WFE formulation (see Eq. (4)):

$$
\mathbf{S}^{i} \boldsymbol{\Phi}_{j}^{i}=\mu_{j}^{i} \boldsymbol{\Phi}_{j}^{i} \quad, \quad\left|\mathbf{S}^{i}-\mu_{j}^{i} \mathbf{I}\right|=0 \quad i=1, \ldots, M .
$$

where $\mathbf{S}^{i}$ is a $\left(2 n^{i} \times 2 n^{i}\right)$ symplectic matrix which is expressed from the dynamic stiffness matrix $\mathbf{D}^{i}$ of uncoupled layer $i$. The reduced local wave mode matrix, say $\widetilde{\boldsymbol{\Phi}}^{i}$, can be typically expressed from the $\left(n^{i} \times 2 m^{i}\right)$ matrices $\widetilde{\boldsymbol{\Phi}}_{\mathrm{q}}^{i}$ and $\widetilde{\boldsymbol{\Phi}}_{\mathrm{F}}^{i}$, reflecting the displacement and force components:

$$
\widetilde{\boldsymbol{\Phi}}^{i}=\left[\begin{array}{c}
\widetilde{\boldsymbol{\Phi}}_{\mathrm{q}}^{i} \\
\widetilde{\boldsymbol{\Phi}}_{\mathrm{F}}^{i}
\end{array}\right] \quad i=1, \ldots, M .
$$

The construction of the global wave mode basis $\left\{\boldsymbol{\Phi}_{j}\right\}_{j}$, from the set of reduced local wave mode bases $\left\{\left\{\widetilde{\boldsymbol{\Phi}}_{j}^{i}\right\}_{j=1, \ldots, 2 m^{i}}\right\}_{i}$, is based on a substructuring scheme which is discussed below.

\subsection{Underlying substructuring scheme}

The substructuring scheme associated with the MWFE formulation is fully presented in ref. [10]. The formalism is briefly recalled hereafter.

The dynamic equilibrium of the set of coupled layers is written

$$
\left[\mathbf{D}^{\text {local }}+\mathbf{D}^{\text {coupling }}\right]\left(\begin{array}{c}
\mathbf{q}^{1} \\
\vdots \\
\mathbf{q}^{M}
\end{array}\right)=\left(\begin{array}{c}
\mathcal{F}^{1} \\
\vdots \\
\mathcal{F}^{M}
\end{array}\right)
$$


where $\mathbf{D}^{\text {local }}$ is a $\left(2 \sum_{i} n^{i} \times 2 \sum_{i} n^{i}\right)$ block diagonal matrix expressing the dynamic stiffness matrices of uncoupled layers and $\mathbf{D}^{\text {coupling }}$ is a $\left(2 \sum_{i} n^{i} \times 2 \sum_{i} n^{i}\right)$ coupling matrix expressing the inertial, elastic and damping forces between layers. $\left\{\mathbf{q}^{i}\right\}_{i}$ are the displacements of the layers; $\mathcal{F}^{i}$ stands for the restriction of the global forces $\mathbf{F}$ to each layer $i$, which is expressed from the forces $\mathbf{F}^{i}$ applied to the uncoupled layer as

$$
\left(\begin{array}{c}
\mathcal{F}^{1} \\
\vdots \\
\mathcal{F}^{M}
\end{array}\right)=\left[\mathbf{I}+\mathbf{E}^{\text {coupling }}\right]\left(\begin{array}{c}
\mathbf{F}^{1} \\
\vdots \\
\mathbf{F}^{M}
\end{array}\right)
$$

The continuity of the forces $\left\{\mathcal{F}^{i}\right\}_{i}$ at coupling interfaces $\left\{\Gamma_{1}\right\}_{i}$ is provided by (15), while the local forces $\left\{\mathbf{F}^{i}\right\}_{i}$ are not necessarily continuous.

Following what was previously presented for the WFE approach (see Section 2.1), the dynamic equilibrium equation (14) of the subsystem is reformulated into a state vector representation of the form (2):

$$
\left(\begin{array}{c}
\mathrm{q}_{\mathrm{R}}^{1} \\
\vdots \\
\frac{\mathrm{q}_{\mathrm{R}}^{M}}{\mathcal{F}_{\mathrm{R}}^{1}} \\
\vdots \\
\mathcal{F}_{\mathrm{R}}^{M}
\end{array}\right)=\mathcal{S}\left(\begin{array}{c}
\mathrm{q}_{\mathrm{L}}^{1} \\
\vdots \\
\frac{\mathrm{q}_{\mathrm{L}}^{M}}{-\mathcal{F}_{\mathrm{L}}^{1}} \\
\vdots \\
-\mathcal{F}_{\mathrm{L}}^{M}
\end{array}\right)
$$

where

$$
\left(\begin{array}{c}
\mathcal{F}_{\mathrm{R}}^{1} \\
\vdots \\
\mathcal{F}_{\mathrm{R}}^{M}
\end{array}\right)=\mathcal{G}^{*}\left(\begin{array}{c}
\mathbf{F}_{\mathrm{R}}^{1} \\
\vdots \\
\mathbf{F}_{\mathrm{R}}^{M}
\end{array}\right) \quad \text { and } \quad\left(\begin{array}{c}
-\mathcal{F}_{\mathrm{L}}^{1} \\
\vdots \\
-\mathcal{F}_{\mathrm{L}}^{M}
\end{array}\right)=\mathcal{G}^{*}\left(\begin{array}{c}
-\mathbf{F}_{\mathrm{L}}^{1} \\
\vdots \\
-\mathbf{F}_{\mathrm{L}}^{M}
\end{array}\right)
$$

The matrices $\mathcal{S}$ and $\mathcal{G}^{*}$ are provided by Eqs. (14) and (15) and are detailed in ref. [10]. $\mathcal{S}$ is a $\left(2 \sum_{i} n^{i} \times 2 \sum_{i} n^{i}\right)$ matrix [10] which is not symplectic a priori. The substructuring scheme provided by Eqs. (16) and (17) is interesting compared to the classic model (see Eq. (2)) as it allows the local kinematic variables (displacements and forces) of each layer cross-section to be considered independently from the surroundings. Specifically, the method seems interesting for constructing the global wave modes of the multi-layered system from a set of local wave mode bases attached to the layers with free interfaces $\left\{\Gamma^{i}\right\}_{i}$ and whose dimensions can be individually tuned to "fit" with each cross-section dynamics. This constitutes the framework of the MWFE formulation presented below. 


\subsection{Construction of the global wave mode basis}

\subsubsection{Global wave mode problem}

Considering the coupling conditions between two consecutive subsystems $k-1$ and $k$, established for each layer $i$ as

$$
\mathbf{q}_{\mathrm{L}}^{i(k)}=\mathbf{q}_{\mathrm{R}}^{i(k-1)} \quad \text { and } \quad-\mathcal{F}_{\mathrm{L}}^{i(k)}=\mathcal{F}_{\mathrm{R}}^{i(k-1)} \quad k=2, \ldots, N \quad i=1, \ldots, M,
$$

and considering, according to Bloch's theorem [12], wave solutions $\left\{\boldsymbol{\Phi}_{j}\right\}_{j}$ of the form (7) in Eq. (16) leads to the following eigenvalue problem

$$
\mathcal{S}\left(\begin{array}{c}
\left(\boldsymbol{\Phi}_{\mathrm{q}}\right)_{j \mid 1} \\
\vdots \\
\frac{\left(\boldsymbol{\Phi}_{\mathrm{q}}\right)_{j \mid M}}{\left(\boldsymbol{\Phi}_{\mathcal{F}}\right)_{j \mid 1}} \\
\vdots \\
\left(\boldsymbol{\Phi}_{\mathcal{F}}\right)_{j \mid M}
\end{array}\right)=\mu_{j}\left(\begin{array}{c}
\left(\boldsymbol{\Phi}_{\mathrm{q}}\right)_{j \mid 1} \\
\vdots \\
\frac{\left(\boldsymbol{\Phi}_{\mathrm{q}}\right)_{j \mid M}}{\left(\boldsymbol{\Phi}_{\mathcal{F}}\right)_{j \mid 1}} \\
\vdots \\
\left(\boldsymbol{\Phi}_{\mathcal{F}}\right)_{j \mid M}
\end{array}\right) \quad, \quad\left|\mathcal{S}-\mu_{j} \mathbf{I}\right|=0
$$

where $\left(\Phi_{\mathrm{q}}\right)_{j \mid i}$ and $\left(\boldsymbol{\Phi}_{\mathcal{F}}\right)_{j \mid i}$ are $\left(n^{i} \times 1\right)$ vectors which represent the restriction of the $(n \times 1)$ global mode components $\left(\boldsymbol{\Phi}_{\mathrm{q}}\right)_{j}$ and $\left(\boldsymbol{\Phi}_{\mathrm{F}}\right)_{j}$ to $S_{\mathrm{L}}^{i}$ or $S_{\mathrm{R}}^{i}$. Furthermore, expressing Eq. (17) in terms of wave mode components allows us to define the set $\left\{\left(\boldsymbol{\Phi}_{\mathrm{F}}\right)_{j \mid i}\right\}_{i}$ as

$$
\left(\begin{array}{c}
\left(\boldsymbol{\Phi}_{\mathcal{F}}\right)_{j \mid 1} \\
\vdots \\
\left(\boldsymbol{\Phi}_{\mathcal{F}}\right)_{j \mid M}
\end{array}\right)=\mathcal{G}^{*}\left(\begin{array}{c}
\left(\boldsymbol{\Phi}_{\mathrm{F}}\right)_{j \mid 1} \\
\vdots \\
\left(\boldsymbol{\Phi}_{\mathrm{F}}\right)_{j \mid M}
\end{array}\right),
$$

which is related to the set of forces $\left\{\mathbf{F}^{i}\right\}_{i}$ applied to the layers with free interfaces. Note that the natures of $\left\{\left\{\left(\boldsymbol{\Phi}_{\mathcal{F}}\right)_{j \mid i}\right\}_{j}\right\}_{i}$ and $\left\{\left\{\left(\boldsymbol{\Phi}_{\mathrm{F}}\right)_{j \mid i}\right\}_{j}\right\}_{i}$ are quite different since they describe the wave force characteristics applied to the coupled and uncoupled layers, respectively. Eq. (20) provides the continuity of $\left\{\left\{\left(\boldsymbol{\Phi}_{\mathcal{F}}\right)_{j \mid i}\right\}_{j}\right\}_{i}$ at $\left\{\Gamma^{i}\right\}_{i}$, which cannot be verified by $\left\{\left\{\left(\boldsymbol{\Phi}_{\mathrm{F}}\right)_{j \mid i}\right\}_{j}\right\}_{i}$ a priori.

Hereafter, the global wave modes $\left\{\boldsymbol{\Phi}_{j}\right\}_{j}$ are constructed from reduced local bases $\left\{\left\{\widetilde{\boldsymbol{\Phi}}_{j}^{i}\right\}_{j=1, \ldots, 2 m^{i}}\right\}_{i}=\left\{\left\{\widetilde{\boldsymbol{\Phi}}_{j}^{1}\right\}_{j},\left\{\widetilde{\boldsymbol{\Phi}}_{k}^{2}\right\}_{k}, \ldots,\left\{\widetilde{\boldsymbol{\Phi}}_{l}^{M}\right\}_{l}\right\}$ attached to the homogeneous uncoupled layers and having specific dimensions $\left\{2 m^{i}\right\}_{i}$. An eigenvalue problem to determine the global wave mode characteristic is then derived.

\subsubsection{MWFE modeling}

This MWFE modeling consists in constructing the global wave modes $\left\{\boldsymbol{\Phi}_{j}\right\}_{j}$ from a set of reduced local bases $\left\{\widetilde{\boldsymbol{\Phi}}^{i}\right\}_{i}$ with specific dimensions $\left\{2 m^{i}\right\}_{i}$, that is:

$$
\left(\boldsymbol{\Phi}_{\mathrm{q}}\right)_{j \mid i}=\sum_{l=1, \ldots, 2 m^{i}} \alpha_{j l}^{i}\left(\widetilde{\boldsymbol{\Phi}}_{\mathrm{q}}^{i}\right)_{l} \quad, \quad\left(\boldsymbol{\Phi}_{\mathrm{F}}\right)_{j \mid i}=\sum_{l=1, \ldots, 2 m^{i}} \alpha_{j l}^{i}\left(\widetilde{\boldsymbol{\Phi}}_{\mathrm{F}}^{i}\right)_{l} \quad i=1, \ldots, M .
$$


The expansion (21) is natural as both global and local wave component sets $\left\{\left(\boldsymbol{\Phi}_{\mathrm{F}}\right)_{j \mid i}\right\}_{i}$ and $\left\{\left\{\left(\Phi_{\mathrm{F}}^{i}\right)_{l}\right\}_{l}\right\}_{i}$ reflect the forces applied to the uncoupled layers. Also notice that the continuity of the global wave force component set $\left\{\left(\Phi_{\mathcal{F}}\right)_{j \mid i}\right\}_{i}$ at coupling interfaces $\left\{\Gamma^{i}\right\}_{i}$ is provided by Eq. (20), which enforces the convergence of the modeling. According to (21) and (20), the construction of the global wave modes $\left\{\boldsymbol{\Phi}_{j}\right\}_{j}$ can be established as follows:

$$
\left(\begin{array}{c}
\left(\boldsymbol{\Phi}_{\mathrm{q}}\right)_{j \mid 1} \\
\vdots \\
\left(\boldsymbol{\Phi}_{\mathrm{q}}\right)_{j \mid M} \\
\hline\left(\boldsymbol{\Phi}_{\mathcal{F}}\right)_{j \mid 1} \\
\vdots \\
\left(\boldsymbol{\Phi}_{\mathcal{F}}\right)_{j \mid M}
\end{array}\right)=\mathcal{B} \boldsymbol{\alpha}_{j}
$$

where $\mathcal{B}$ is given by

$$
\mathcal{B}=\left[\begin{array}{cc}
\mathbf{I} & \mathbf{0} \\
\mathbf{0} & \mathcal{G}^{*}
\end{array}\right]\left[\begin{array}{c}
\widetilde{\boldsymbol{\Phi}}_{\mathrm{q}}^{\text {local }} \\
\widetilde{\boldsymbol{\Phi}}_{\mathrm{F}}^{\text {local }}
\end{array}\right]
$$

and expressed from the following $\left(\sum_{i} n^{i} \times 2 \sum_{i} m^{i}\right)$ matrices $\widetilde{\boldsymbol{\Phi}}_{\mathrm{q}}^{\text {local }}$ and $\widetilde{\boldsymbol{\Phi}}_{\mathrm{F}}^{\text {local }}$

$$
\widetilde{\boldsymbol{\Phi}}_{\mathrm{q}}^{\text {local }}=\left[\begin{array}{cccc}
\widetilde{\boldsymbol{\Phi}}_{\mathrm{q}}^{1} & \mathbf{0} & \ldots & \mathbf{0} \\
\mathbf{0} & \widetilde{\boldsymbol{\Phi}}_{\mathrm{q}}^{2} & \ldots & \mathbf{0} \\
\vdots & \vdots & \ddots & \vdots \\
\mathbf{0} & \mathbf{0} & \ldots & \widetilde{\boldsymbol{\Phi}}_{\mathrm{q}}^{M}
\end{array}\right] \quad \text { and } \quad \widetilde{\boldsymbol{\Phi}}_{\mathrm{F}}^{\text {local }}=\left[\begin{array}{cccc}
\widetilde{\boldsymbol{\Phi}}_{\mathrm{F}}^{1} & \mathbf{0} & \ldots & \mathbf{0} \\
\mathbf{0} & \widetilde{\boldsymbol{\Phi}}_{\mathrm{F}}^{2} & \ldots & \mathbf{0} \\
\vdots & \vdots & \ddots & \vdots \\
\mathbf{0} & \mathbf{0} & \ldots & \widetilde{\boldsymbol{\Phi}}_{\mathrm{F}}^{M}
\end{array}\right]
$$

In Eq. (22), $\left\{\boldsymbol{\alpha}_{j}\right\}_{j}$ stands for the set of $\left(2 \sum_{i} m^{i} \times 1\right)$ generalized coordinate vectors,

$$
\boldsymbol{\alpha}_{j}=\left[\begin{array}{c}
\boldsymbol{\alpha}_{j}^{1} \\
\boldsymbol{\alpha}_{j}^{2} \\
\vdots \\
\boldsymbol{\alpha}_{j}^{M}
\end{array}\right] \quad, \quad \boldsymbol{\alpha}_{j}^{i}=\left[\begin{array}{c}
\alpha_{j 1}^{i} \\
\alpha_{j 2}^{i} \\
\vdots \\
\alpha_{j m^{i}}^{i}
\end{array}\right] \quad i=1, \ldots, M
$$

which has to be determined in the MWFE context by means of the substructuring scheme described earlier. The methodology is described as follows: Inserting Eq. (22) into eigenvalue problem (19) results in the following $\left(2 \sum_{i} n^{i} \times 2 \sum_{i} m^{i}\right)$ overdetermined system:

$$
\mathcal{S B} \boldsymbol{\alpha}_{j}=\mu_{j} \mathcal{B} \boldsymbol{\alpha}_{j}
$$

which can be reduced to a square $\left(2 \sum_{i} m^{i} \times 2 \sum_{i} m^{i}\right)$ when it is multiplied by $\mathcal{B}^{T} \mathbf{J}$, that is

$$
\mathcal{B}^{T} \mathbf{J S B} \boldsymbol{\alpha}_{j}=\mu_{j} \mathcal{B}^{T} \mathbf{J} \mathcal{B} \boldsymbol{\alpha}_{j}
$$


where

$$
\mathbf{J}=\left[\begin{array}{cc}
\mathbf{0} & \mathbf{I}_{\left(2 \sum_{i} n^{i}\right)} \\
-\mathbf{I}_{\left(2 \sum_{i} n^{i}\right)} & \mathbf{0}
\end{array}\right]
$$

The problem is supposed to be well-conditioned because (i) it is homogeneous and not partitioned into displacement and force components, (ii) the local wave mode matrices $\left\{\widetilde{\boldsymbol{\Phi}}^{i}\right\}_{i}$ are supposed to be normalized by means of the adimensioned Hermitian norm [10]. The set of modal participations $\left\{\boldsymbol{\alpha}_{j}\right\}_{j}$ can be evaluated by solving a classic eigenvalue problem

$$
\mathbf{S}^{\mathrm{MWFE}} \boldsymbol{\alpha}_{j}=\mu_{j} \boldsymbol{\alpha}_{j} \quad, \quad\left|\mathbf{S}^{\mathrm{MWFE}}-\mu_{j} \mathbf{I}\right|=0,
$$

where

$$
\mathrm{S}^{\mathrm{MWFE}}=\left[\mathcal{B}^{T} \mathbf{J} \mathcal{B}\right]^{-1} \mathcal{B}^{T} \mathbf{J S B}
$$

The invertibility of the matrix $\left[\mathcal{B}^{T} \mathbf{J} \mathcal{B}\right]$, which appears on the right hand side of Eq. (30), can be verified if the matrix $\mathcal{B}$ does not contain dependent vectors: this is presumed to be true when the dimension of each local basis $\left\{\widetilde{\boldsymbol{\Phi}}_{j}^{i}\right\}_{j}$ does not overestimate the dynamics of each layer $i$.

The eigenvalue problem (29) is central to the MWFE formulation. Given a set of reduced local bases $\left\{\left\{\widetilde{\boldsymbol{\Phi}}_{j}^{i}\right\}_{j}\right\}_{i}$ having appropriate dimensions, the formulation consists in finding the eigenvalues $\left\{\mu_{j}\right\}_{j}$, which describe the global wave mode velocities, and the eigenvectors $\left\{\boldsymbol{\alpha}_{j}\right\}_{j}$, providing the restrictions of the global wave mode shapes $\left\{\left\{\boldsymbol{\Phi}_{j \mid i}\right\}_{i}\right\}_{j}$ to the surfaces $\left\{S_{\mathrm{L}}^{i}\right\}_{i}$ or $\left\{S_{\mathrm{R}}^{i}\right\}_{i}$ by means of Eq. (22).

The advantages of the MWFE formulation compared to the WFE formulation are:

- The size of the eigenvalue problem (29) corresponds to a sum of dimensions which exactly capture the dynamics of the layer cross-section: dependency among eigenvectors is removed and the essential behavior is captured;

- The size of the eigenvalue problem (29) is generally smaller than the classic eigenvalue problem (4) (see Section 2.3), allowing a reduction of the numerical cost;

- All the components of $\mathbf{S}^{\mathrm{MWFE}}$ are homogeneous and are not partitioned into displacement and force components: the problem of the ill-conditioned classic operator $\mathbf{S}$ is removed, a priori. 


\section{Forced response calculation}

\subsection{Generality}

In the MWFE framework, the kinematic variables can be defined as:

$$
\mathcal{U}_{\mathrm{L}}=\left(\begin{array}{c}
\mathrm{q}_{\mathrm{L}}^{1} \\
\vdots \\
\mathrm{q}_{\mathrm{L}}^{M} \\
\hline-\mathcal{F}_{\mathrm{L}}^{1} \\
\vdots \\
-\mathcal{F}_{\mathrm{L}}^{M}
\end{array}\right) \quad, \quad \mathcal{U}_{\mathrm{R}}=\left(\begin{array}{c}
\mathrm{q}_{\mathrm{R}}^{1} \\
\vdots \\
\frac{\mathrm{q}_{\mathrm{R}}^{M}}{\mathcal{F}_{\mathrm{R}}^{1}} \\
\vdots \\
\mathcal{F}_{\mathrm{R}}^{M}
\end{array}\right)
$$

This leads to a description of the global system quite similar to Eq. (6), that is

$$
\mathcal{U}_{\mathrm{L}}^{(k)}=\mathcal{S U}_{\mathrm{L}}^{(k-1)} \quad, \quad \mathcal{U}_{\mathrm{R}}^{(k)}=\mathcal{S U}_{\mathrm{R}}^{(k-1)} \quad k=2, \ldots, N
$$

Also, let $\left\{\boldsymbol{\Phi}_{j}^{\mathrm{MWFE}}\right\}_{j}$ denote the global modes obtained via the MWFE method, that is

$$
\boldsymbol{\Phi}_{j}^{\mathrm{MWFE}}=\left(\begin{array}{c}
\left(\boldsymbol{\Phi}_{\mathrm{q}}\right)_{j \mid 1} \\
\vdots \\
\left(\boldsymbol{\Phi}_{\mathrm{q}}\right)_{j \mid M} \\
\left(\boldsymbol{\Phi}_{\mathcal{F}}\right)_{j \mid 1} \\
\vdots \\
\left(\boldsymbol{\Phi}_{\mathcal{F}}\right)_{j \mid M}
\end{array}\right) \quad \text { such that } \quad \boldsymbol{\Phi}_{j}^{\mathrm{MWFE}}=\mathcal{B} \boldsymbol{\alpha}_{j} \quad j=1, \ldots, 2 \sum_{i} m^{i}
$$

The framework of the MWFE model consists in expanding the state vectors $\mathcal{U}_{\mathrm{L}}$ and $\mathcal{U}_{\mathrm{R}}$ onto the global wave mode basis constructed via the formalism, that is

$$
\mathcal{U}_{\mathrm{L}}^{(k)}=\left[\widetilde{\boldsymbol{\Phi}}^{\mathrm{MWFE}}\right] \widetilde{\mathbf{Q}}^{(k)} \quad, \quad \mathcal{U}_{\mathrm{R}}^{(k)}=\left[\widetilde{\boldsymbol{\Phi}}^{\mathrm{MWFE}}\right] \widetilde{\mathbf{Q}}^{(k+1)} \quad k=1, \ldots, N,
$$

where $\left[\widetilde{\boldsymbol{\Phi}}^{\text {MWFE }}\right]$ denotes the matrix of the relevant LF and MF global modes extracted from the full $\left(2 \sum_{i} n^{i} \times 2 \sum_{i} m^{i}\right)$ wave mode matrix $\left[\boldsymbol{\Phi}^{\mathrm{MWFE}}\right]$ which is obtained via the MWFE formulation. Partitioning $\left[\widetilde{\Phi}^{M W F E}\right]$ into displacement and forces components, as well as incident and reflected wave components, gives

$$
\left[\widetilde{\boldsymbol{\Phi}}^{\mathrm{MWFE}}\right]=\left[\begin{array}{cc}
{\left[\widetilde{\boldsymbol{\Phi}}^{\mathrm{MWFE}}\right]_{\mathbf{q}}^{\mathrm{inc}}} & {\left[\widetilde{\boldsymbol{\Phi}}^{\mathrm{MWFE}}\right]_{\mathbf{q}}^{\text {ref }}} \\
{\left[\widetilde{\boldsymbol{\Phi}}^{\mathrm{MWFE}}\right]_{\mathbf{F}}^{\text {inc }}} & {\left[\widetilde{\boldsymbol{\Phi}}^{\mathrm{MWFE}}\right]_{\mathbf{F}}^{\text {ref }}}
\end{array}\right],
$$

where $\left[\widetilde{\boldsymbol{\Phi}}^{\text {MWFE }}\right]_{\mathbf{q}}^{\text {inc }},\left[\widetilde{\boldsymbol{\Phi}}^{\text {MWFE }}\right]_{\mathbf{F}}^{\text {inc }},\left[\widetilde{\boldsymbol{\Phi}}^{\text {MWFE }}\right]_{\mathbf{q}}^{\text {ref }}$ and $\left[\widetilde{\boldsymbol{\Phi}}^{\text {MWFE }}\right]_{\mathbf{F}}^{\text {ref }}$ are matrices of the same dimension; the subscripts $\mathbf{q}$ and $\mathbf{F}$ refer to the displacement and force components, respectively. Here, the notations inc and ref provide a local description of the system and 
denote the waves which are incident to - and reflected by - a specific boundary, respectively. In theory, the matrices $\left[\widetilde{\boldsymbol{\Phi}}^{\mathrm{MWFE}}\right]_{\mathbf{q}}^{\text {inc }},\left[\widetilde{\boldsymbol{\Phi}}^{\mathrm{MWFE}}\right]_{\mathbf{F}}^{\text {inc }},\left[\widetilde{\boldsymbol{\Phi}}^{\mathrm{MWFE}}\right]_{\mathbf{q}}^{\text {ref }}$ and $\left[\widetilde{\boldsymbol{\Phi}}^{\mathrm{MWFE}}\right]_{\mathbf{F}}^{\text {ref }}$ are linked through the following relationship

$$
\left[\widetilde{\boldsymbol{\Phi}}^{\mathrm{MWFE}}\right]_{\mathbf{q}}^{\mathrm{inc}}=\left[\widetilde{\boldsymbol{\Phi}}^{\mathrm{MWFE}}\right]_{\mathbf{q}}^{\mathrm{ref}} \quad, \quad\left[\widetilde{\boldsymbol{\Phi}}^{\mathrm{MWFE}}\right]_{\mathbf{F}}^{\mathrm{inc}}=-\left[\widetilde{\boldsymbol{\Phi}}^{\mathrm{MWFE}}\right]_{\mathbf{F}}^{\mathrm{ref}} .
$$

Also, the diagonal eigenvalue matrix $\widetilde{\boldsymbol{\mu}}^{\text {inc }}$ of the incident modes are linked to the diagonal eigenvalue matrix $\widetilde{\boldsymbol{\mu}}^{\text {ref }}$ of the reflected modes through the following relationship

$$
\widetilde{\boldsymbol{\mu}}^{\text {inc }}=\left(\widetilde{\boldsymbol{\mu}}^{\mathrm{ref}}\right)^{-1}=\widetilde{\boldsymbol{\mu}} .
$$

Eqs. (36) and (37) are provided by the state vector representation (31) and will be used to enforce the duality between incident and reflected waves, which is not perfectly verified in practice as numerical dispersion effects can occur. In brief, the wave mode extraction process consists for instance in calculating incident modes and characterizing the reflected modes from Eqs. (36) and (37).

Invoking (32) and (37) into (34) provides the governing equation of the system

$$
\widetilde{\mathbf{Q}}^{\text {inc }(k)}=\widetilde{\boldsymbol{\mu}}^{k-1} \widetilde{\mathbf{Q}}^{\text {inc }(1)} \quad, \quad \widetilde{\mathbf{Q}}^{\mathrm{ref}(k)}=\widetilde{\boldsymbol{\mu}}^{-(k-1)} \widetilde{\mathbf{Q}}^{\mathrm{ref}(1)} \quad k=1, \ldots, N+1,
$$

where $\widetilde{\mathrm{Q}}^{\text {inc }}$ and $\widetilde{\mathrm{Q}}^{\text {ref }}$ are the amplitudes of the incident and reflected modes, respectively. Eq. (38) provides the spatial distribution of the modal amplitudes along the system from $\widetilde{\mathrm{Q}}^{\text {inc(1) }}$ and $\widetilde{\mathrm{Q}}^{\text {ref(1)}}$ which are expressed from the boundary conditions. For instance, Dirichlet and Neumann boundary conditions write $\mathbf{q}=\mathbf{q}_{0}$ and $\mathbf{F}=\mathbf{F}_{0}$ which, in state vector representation results in

$$
\begin{aligned}
& {[\mathbf{I} \mid \mathbf{0}] \mathcal{U}=\mathcal{L} \mathbf{q}_{0} \quad \text { (Dirichlet condition) }} \\
& {[\mathbf{0} \mid \mathbf{I}] \mathcal{U}= \pm \mathcal{L} \mathbf{F}_{0} \quad \text { (Neumann condition) }}
\end{aligned}
$$

Here, $\mathcal{L}$ is a Boolean operator which links the DOFs of each layer cross-section to the DOFs of the global cross-section, that is

$$
\left(\begin{array}{c}
\mathbf{q}^{1} \\
\vdots \\
\mathbf{q}^{M}
\end{array}\right)=\mathcal{L} \mathbf{q} \quad, \quad\left(\begin{array}{c}
\mathcal{F}^{1} \\
\vdots \\
\mathcal{F}^{M}
\end{array}\right)=\mathcal{L} \mathbf{F} .
$$

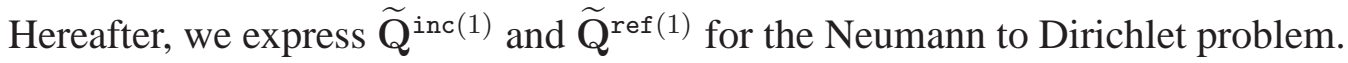

\subsection{The Neumann to Dirichlet problem}

We consider a force field $\mathbf{F}_{0}$ and a displacement field $\mathbf{q}_{0}$ applied to the left and right ends of the system, respectively. The Neumann to Dirichlet problem can be stated as follows:

For $k \in\{1, \ldots, N+1\}$, find $\mathrm{Q}^{\text {inc }(k)}$ and $\mathrm{Q}^{\text {ref(k) }}$ such that

$$
\widetilde{\mathbf{Q}}^{\text {inc }(k)}=\widetilde{\boldsymbol{\mu}}^{k-1} \widetilde{\mathbf{Q}}^{\text {inc }(1)} \quad, \quad \widetilde{\mathbf{Q}}^{\mathrm{ref}(k)}=\widetilde{\boldsymbol{\mu}}^{-(k-1)} \widetilde{\mathbf{Q}}^{\mathrm{ref}(1)}
$$


and

$$
\begin{aligned}
& {[\mathbf{0} \mid \mathbf{I}] \mathcal{U}_{\mathrm{L}}^{1}=-\mathcal{L} \mathbf{F}_{0}} \\
& {[\mathbf{I} \mid \mathbf{0}] \mathcal{U}_{\mathrm{R}}^{N}=\mathcal{L} \mathbf{q}_{0}}
\end{aligned}
$$

The boundary conditions are provided by (42). Invoking (34), (35) and (36), those conditions are expanded on the wave basis to give

$$
\begin{aligned}
& {\left[\widetilde{\boldsymbol{\Phi}}^{\mathrm{MWFE}}\right]_{\mathbf{F}}^{\text {inc }} \widetilde{\mathbf{Q}}^{\text {inc }(1)}-\left[\widetilde{\boldsymbol{\Phi}}^{\mathrm{MWFE}}\right]_{\mathbf{F}}^{\text {inc }} \widetilde{\mathbf{Q}}^{\mathrm{ref}(1)}=-\mathcal{L} \mathbf{F}_{0},} \\
& {\left[\widetilde{\boldsymbol{\Phi}}^{\mathrm{MWFE}}\right]_{\mathbf{q}}^{\text {inc }} \widetilde{\mathbf{Q}}^{\text {inc }(N+1)}+\left[\widetilde{\boldsymbol{\Phi}}^{\mathrm{MWFE}}\right]_{\mathbf{q}}^{\text {inc }} \widetilde{\mathbf{Q}}^{\mathrm{ref}(N+1)}=\mathcal{L} \mathbf{q}_{0},}
\end{aligned}
$$

According to (41), (43) gives

$$
\begin{aligned}
& {\left[\widetilde{\boldsymbol{\Phi}}^{\mathrm{MWFE}}\right]_{\mathbf{F}}^{\text {inc }} \widetilde{\mathbf{Q}}^{\text {inc }(1)}-\left[\widetilde{\boldsymbol{\Phi}}^{\mathrm{MWFE}}\right]_{\mathbf{F}}^{\text {inc }} \widetilde{\mathbf{Q}}^{\mathrm{ref}(1)}=-\mathcal{L} \mathbf{F}_{0},} \\
& {\left[\widetilde{\boldsymbol{\Phi}}^{\mathrm{MWFE}}\right]_{\mathbf{q}}^{\text {inc }} \widetilde{\boldsymbol{\mu}}^{N} \mathbf{Q}^{\text {inc }(1)}+\left[\widetilde{\boldsymbol{\Phi}}^{\mathrm{MWFE}}\right]_{\mathbf{q}}^{\text {inc }} \widetilde{\boldsymbol{\mu}}^{-N} \widetilde{\mathbf{Q}}^{\text {ref }(1)}=\mathcal{L} \mathbf{q}_{0},}
\end{aligned}
$$

This leads to the following matrix form

$$
\left[\begin{array}{cc}
\mathbf{I} & -\mathbf{I} \\
\widetilde{\boldsymbol{\mu}}^{N} & \widetilde{\boldsymbol{\mu}}^{-N}
\end{array}\right]\left(\begin{array}{c}
\widetilde{\mathbf{Q}}^{\text {inc }(1)} \\
\widetilde{\mathbf{Q}}^{\text {ref }(1)}
\end{array}\right)=\left(\begin{array}{c}
-\left(\left[\widetilde{\boldsymbol{\Phi}}^{\text {MWFE }}\right]_{\mathbf{F}}^{\text {inc }}\right)^{+} \mathcal{L} \mathbf{F}_{0} \\
\left(\left[\widetilde{\boldsymbol{\Phi}}^{\text {MWFE }}\right]_{\mathbf{q}}^{\text {inc }}\right)^{+} \mathcal{L} \mathbf{q}_{0}
\end{array}\right),
$$

where + denotes the left pseudo-inverse. The matrix on the left hand side of (45) appears badly conditioned as the ratio between the maximum value of $\widetilde{\boldsymbol{\mu}}^{-N}$ and the minimum value of $\widetilde{\boldsymbol{\mu}}^{N}$, or vice versa, can be extremely large. Without loss of generality, $\|\tilde{\boldsymbol{\mu}}\|_{\max }<1$ which can be verified for modes propagating towards the right end of the system [11]. In this sense, the system (45) can be rewritten to a more convenient way to give

$$
\left[\begin{array}{cc}
\mathbf{I} & -\widetilde{\boldsymbol{\mu}}^{N} \\
\widetilde{\boldsymbol{\mu}}^{N} & \mathbf{I}
\end{array}\right]\left[\begin{array}{cc}
\mathbf{I} & \mathbf{0} \\
\mathbf{0} & \widetilde{\boldsymbol{\mu}}^{-N}
\end{array}\right]\left(\begin{array}{c}
\widetilde{\mathbf{Q}}^{\text {inc }(1)} \\
\widetilde{\mathbf{Q}}^{\text {ref }(1)}
\end{array}\right)=\left(\begin{array}{c}
-\left(\left[\widetilde{\boldsymbol{\Phi}}^{\mathrm{MWFE}}\right]_{\mathbf{F}}^{\text {inc }}\right)^{+} \mathcal{L} \mathbf{F}_{0} \\
\left(\left[\widetilde{\boldsymbol{\Phi}}^{\mathrm{MWFE}}\right]_{\mathbf{q}}^{\text {inc }}\right)^{+} \mathcal{L} \mathbf{L}_{0}
\end{array}\right) .
$$

Here, the first matrix on the left hand side of (46) is a priori well conditioned; the second matrix on the left hand side of (46) is diagonal and can be inverted without difficulties. The resolution of (46) gives

$$
\left(\begin{array}{c}
\widetilde{\mathbf{Q}}^{\text {inc }(1)} \\
\widetilde{\mathbf{Q}}^{\text {ref }(1)}
\end{array}\right)=\left[\begin{array}{cc}
\mathbf{I} & \mathbf{0} \\
\mathbf{0} & \widetilde{\boldsymbol{\mu}}^{N}
\end{array}\right]\left[\begin{array}{cc}
\mathbf{I} & -\widetilde{\boldsymbol{\mu}}^{N} \\
\widetilde{\boldsymbol{\mu}}^{N} & \mathbf{I}
\end{array}\right]^{-1}\left(\begin{array}{c}
-\left(\left[\widetilde{\boldsymbol{\Phi}}^{\mathrm{MWFE}}\right]_{\mathbf{F}}^{\text {inc }}\right)^{+} \mathcal{L} \mathbf{F}_{0} \\
\left(\left[\widetilde{\boldsymbol{\Phi}}^{\mathrm{MWFE}}\right]_{\mathbf{q}}^{\text {inc }}\right)^{+} \mathcal{L} \mathbf{q}_{0}
\end{array}\right) .
$$

Eq. (47) provides the modal amplitudes $\mathrm{Q}^{\mathrm{inc}(1)}$ and $\mathrm{Q}^{\mathrm{ref}(1)}$ at the left end of the system. This gives from (41) the spatial distribution of the modal amplitudes along the system, and provides from (34) its dynamical behavior. Numerical verification of the MWFE model provided by (47) is the main object of the following section. 


\section{Numerical simulations}

The vibratory behavior of a sandwich beam, consisting in a soft core surrounded by two stiff skins, is numerically evaluated. The characteristics of a typical subsystem of the structure are similar to those depicted in Section 2.3. The global length of the beam is $0.4 \mathrm{~m}$ and is generated using $N=200$ subsystems. The system is illustrated in Figure 5: it is clamped at the right end over the global cross-section and excited at the left end over the bottom skin cross-section. Axial and transverse loads, as depicted in Figure 5, are individually studied. Those loads numerically describe surface force fields applied over the skin cross-section uniformly.

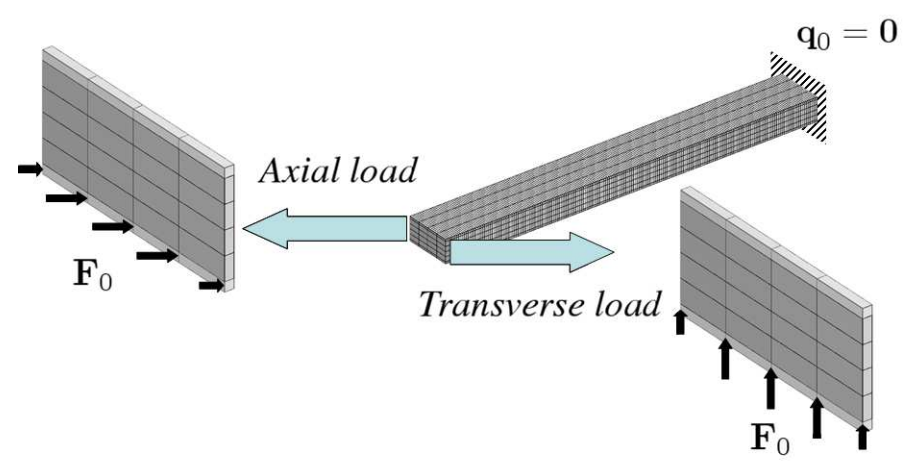

Figure 5: Finite element model of the global system, whose right end is clamped and whose bottom part of the left end is excited by axial or transverse load.

The solutions provided by the MWFE and WFE formulations are compared together and are compared with standard FE simulation in the frequency band $\mathcal{B}_{f}=$ $[100 \mathrm{~Hz}, 1500 \mathrm{~Hz}]$. The MWFE solutions are provided by Eq. (47) while the WFE solutions are provided by the alternative system

$$
\left(\begin{array}{c}
\widetilde{\mathbf{Q}}^{\text {inc }(1)} \\
\widetilde{\mathbf{Q}}^{\text {ref }(1)}
\end{array}\right)=\left[\begin{array}{cc}
\mathbf{I} & \mathbf{0} \\
\mathbf{0} & \widetilde{\boldsymbol{\mu}}^{N}
\end{array}\right]\left[\begin{array}{cc}
\mathbf{I} & -\widetilde{\boldsymbol{\mu}}^{N} \\
\widetilde{\boldsymbol{\mu}}^{N} & \mathbf{I}
\end{array}\right]^{-1}\left(\begin{array}{c}
-\left(\widetilde{\boldsymbol{\Phi}}_{\mathbf{F}}^{\text {inc }}\right)^{+} \mathbf{F}_{0} \\
\left(\widetilde{\boldsymbol{\Phi}}_{\mathbf{q}}^{\text {inc }}\right)^{+} \mathbf{q}_{0}
\end{array}\right),
$$

where $\widetilde{\Phi}$ denotes the matrix of the global modes obtained via WFE.

\subsection{Construction of the global modes using the MWFE formula- tion}

In the MWFE framework, the global modes are constructed from the local modes of the layers. Those modes are supposed to significantly contribute to the dynamics of the system within $\mathcal{B}_{f}$. For each layer (skin and core), the local wave basis contains the classic propagating and evanescent modes and the cross-section modes which cut on or are close to cut on within $\mathcal{B}_{f}$. In the present case, the numbers of local modes 
are $m^{1}=m^{3}=6$ for the skins and $m^{2}=30$ for the core, so that the dimension of the MWFE problem is $2\left(m^{1}+m^{2}+m^{3}\right)=84$ and appears significantly smaller than the dimension of the WFE problem, say $2 n=210$. The global modes are obtained from eigenvalue problem (29). Four relevant propagating modes - say longitudinal, shearing, propagating flexural and evanescent flexural - with regard to the type of excitation which is considered, are described in Figure 6 at $100 \mathrm{~Hz}$.

(a)

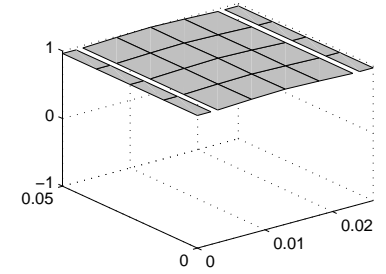

(c)

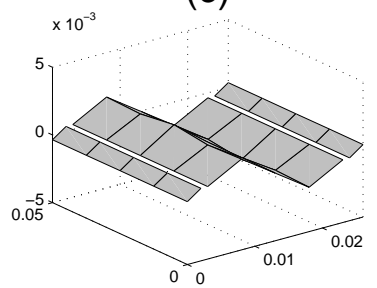

(b)

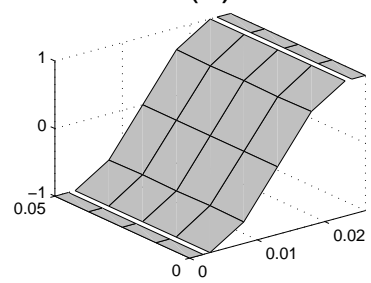

(d)

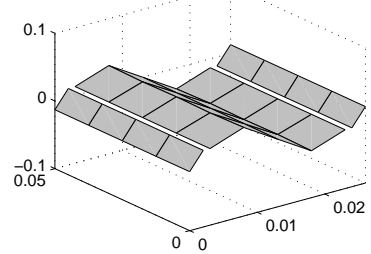

Figure 6: Global wave mode shapes (real parts of the displacement along the axis $x$ ) obtained from the MWFE formulation at $100 \mathrm{~Hz}$ : (a) longitudinal; (b) shearing; (c) propagating flexural; (d) evanescent flexural.

\subsection{Dynamic behavior of the system under axial load}

The sandwich structure is excited at the left end by an axial load ( $x$-direction) over the bottom skin cross-section (see Figure 5). The dynamic response of the left end in the $x$ - direction, at the center of the bottom skin cross-section, is numerically evaluated using standard FE, WFE and MWFE.

The WFE and MWFE formulations are first compared when only 4 global modes are used to predict the dynamics of the system. The results are provided in Figure 7. The lack of convergence of the WFE formulation is clearly established above $600 \mathrm{~Hz}$. This is explained because each wave mode solution obtained via WFE reveals dissimilar natures over $\mathcal{B}_{f}$. Changes into wave motion natures are inherent to the WFE formulation and are caused by wave mode dependency [10]. The LF behavior of the longitudinal mode is omitted in the high frequency range, so that the wave mode basis is not consistent to describe the dynamics of the system. On the other hand, the MWFE solution is coherent with FE simulations within $\mathcal{B}_{f}$. The formulation provides the first two principal resonances of the system and provides a global estimate of the vibratory levels above $1000 \mathrm{~Hz}$. It is to be emphasized that the MWFE formulation slightly overestimates the resonance frequencies of the system as it generally overestimates the wave velocities. Those pollution effects may be related to the density of 
the cross-section mesh and/or to the size of the local wave mode bases.
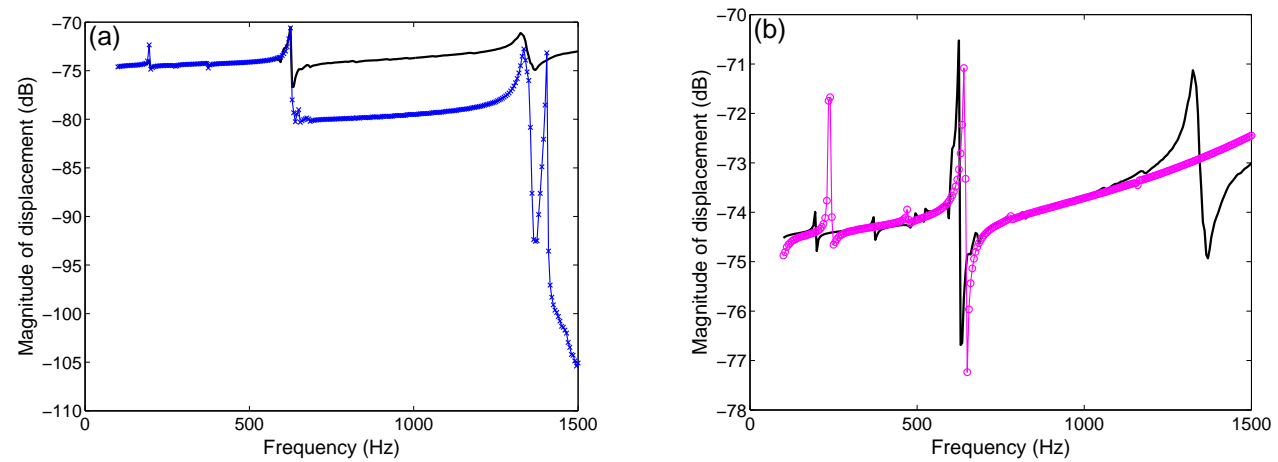

Figure 7: Frequency response of the sandwich beam under axial load: (a) WFE with 4 modes $(-\mathrm{X}-)$ compared to FE (-); (b) MWFE with 4 modes $(-\mathrm{O}-)$ compared to FE $(-)$.

In a second step, we have applied the WFE formulation using 40 significant LF and MF modes. The WFE wave basis contains the classic propagating modes as well as the cross-section modes which cut on or are close to cut on within $\mathcal{B}_{f}$, so that the basis is supposed to be sufficiently rich to accurately provide the required dynamics. Also, we have applied the MWFE formulation using 10 significant propagating and cutting-on cross-section modes. The results are provided in Figure 8. Here again, the WFE formulation suffers a lack of convergence. This is explained as LF classic wave motions are omitted by the formulation as spurious high order solutions are preferentially selected. On the other hand, the MWFE formulation provides an estimate of the dynamic behavior of the system below $1000 \mathrm{~Hz}$ and predicts additional resonances around $700 \mathrm{~Hz}$. High order resonances are not predicted by the formulation as it describes global modes which are consistent with the dimension of the local bases. In this study, those bases are supposed to principally support LF behavior.
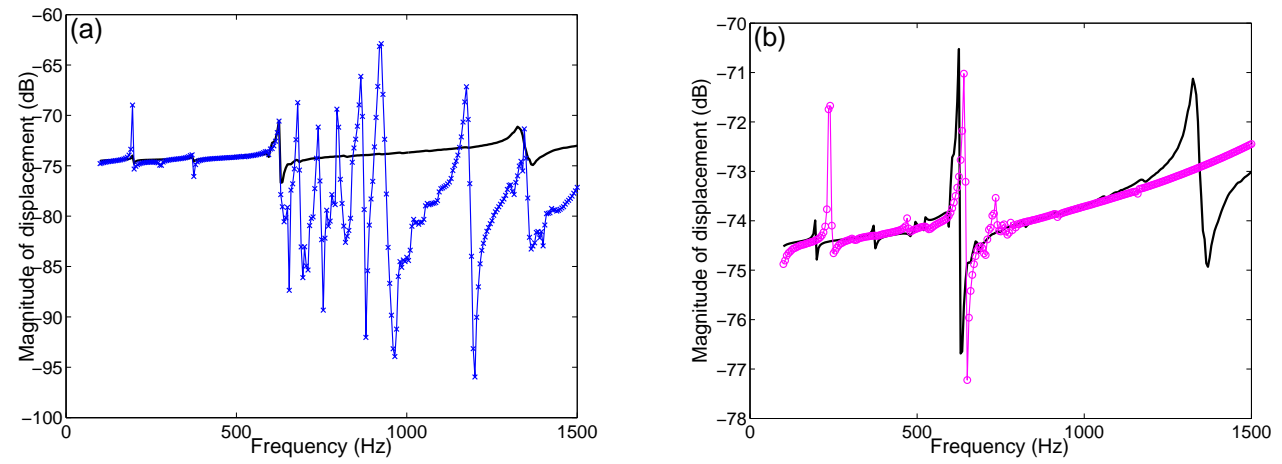

Figure 8: Frequency response of the sandwich beam under axial load: (a) WFE with 40 modes $(-\mathrm{X}-)$ compared to FE (-); (b) MWFE with 10 modes $(-\mathrm{O}-)$ compared to FE $(-)$. 


\subsection{Dynamic behavior of the system under transverse load}

Here, the sandwich structure is excited at the left end by a transverse load ( $z$-direction) over the bottom skin cross-section (see Figure 5). The dynamic response of the left end in the $z$ - direction, at the center of the bottom skin cross-section, is numerically evaluated using standard FE, WFE and MWFE.

Here again, the WFE and MWFE formulations have been compared with standard FE simulations. The results provided by the wave formulations using 4 significant modes are given in Figure 9. Both WFE and MWFE formulations suffer a lack of convergence. The WFE formulation accurately predicts five resonances below $1100 \mathrm{~Hz}$ which may be attributed to LF as well as MF wave motions. However, the method provides erroneous solutions above $1100 \mathrm{~Hz}$ as the dynamics of the global system cannot be supported by LF wave motions. The MWFE formulation provides a global estimate of the vibratory levels of the system over $\mathcal{B}_{f}$. However, its convergence to describe the resonance of the system appears weaker than WFE. The results provided by WFE and MWFE, when 40 and 10 modes are respectively used, are given in Figure 10. The WFE formulation accurately describes the behavior of the system within $\mathcal{B}_{f}$ : this is explained because the wave mode basis contains a sufficient number, say 40, of LF and MF modes. On the other hand, the MWFE formulation generally provides the structural resonance frequencies below $1200 \mathrm{~Hz}$ when 10 global modes are used only.
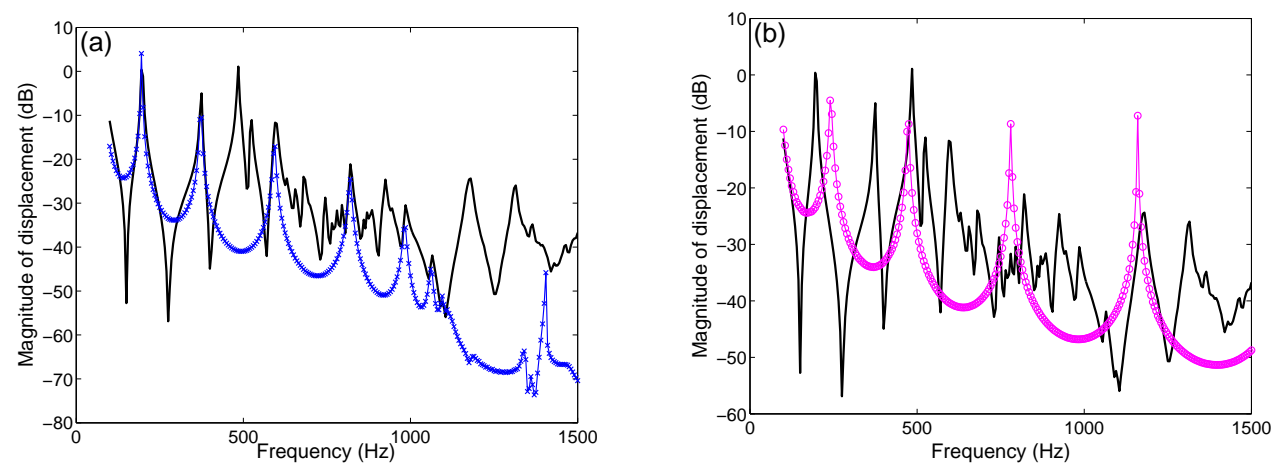

Figure 9: Frequency response of the sandwich beam under transverse load: (a) WFE with 4 modes $(-\mathrm{x}-)$ compared to FE (-); (b) MWFE with 4 modes (-O-) compared to FE (-).

\subsection{Discussions}

We have applied the WFE and MWFE formulations to describe the forced response of a sandwich structure. It is to be emphasized that WFE and MWFE methods provide large numerical cost savings compared to standard FE simulations. The lack of convergence of WFE has been clearly established when axial excitation is considered. The WFE method also involves large size wave bases to correctly capture the LF structural motions. This can be justified with regard to the varying frequency behavior of each WFE mode. On the other hand, the MWFE formulation successfully describes 

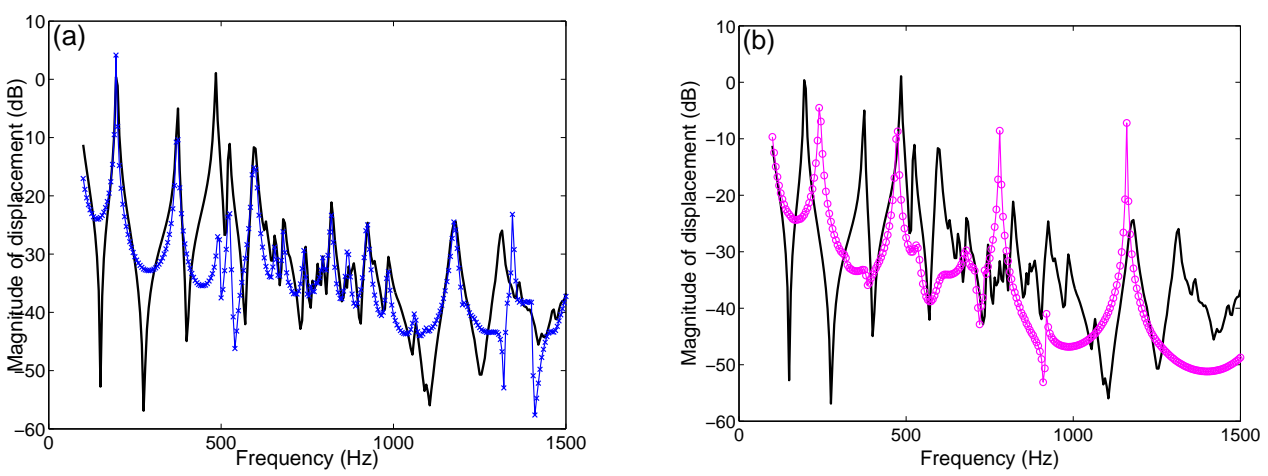

Figure 10: Frequency response of the sandwich beam under transverse load: (a) WFE with 40 modes $(-\mathrm{X}-)$ compared to FE (-); (b) MWFE with 10 modes $(-\mathrm{O}-)$ compared to FE $(-)$.

the dynamics of the sandwich structure within $[100 \mathrm{~Hz}, 1200 \mathrm{~Hz}]$ when 10 modes are used only. This is explained in particular because the formulation correctly provides the LF wave motions within $\mathcal{B}_{f}$. The lack of convergence of the method may be related to the size of the local wave mode bases to construct the global modes and which should ideally fit with the local cross-section dynamics at each frequency step.

\section{Conclusion}

The wave propagation in multi-layered elastic systems has been numerically studied. It was emphasized, when multiscale behavior between layers is considered, that the wave finite element (WFE) formulation is inefficient to capture the essential global wave motion behavior. To solve this issue, a dynamic subtructuring scheme, allowing the global wave modes of systems to be constructed from a set of reduced local wave mode bases, has been proposed. The formulation, termed modified wave finite element (MWFE), allows the classic wave motions to be correctly captured. We have applied the MWFE formulation to describe the forced response of multi-layered systems and we have presented the related Neumann to Dirichlet problem. Numerical WFE and MWFE simulations involving a sandwich structure under axial and transverse loads have been proceeded. The lack of convergence of the WFE formulation to describe the response of the axially loaded structure has been emphasized. The MWFE formulation provides the resonance frequencies of the system in the LF domain and provides a global estimate of the vibratory levels at higher frequencies. The formulation suffers from slight numerical pollution effects, a priori attributed to the size of the local wave mode bases chosen for global mode construction, which could be investigated in further works. 


\section{References}

[1] O. C. Zienkiewicz and R. L. Taylor. The Finite Element Method (first volume). Butterworth-Heinemann, fifth edition, Oxford, 2000.

[2] I. Babuska and T. Strouboulis. The Finite Element Method and Its Reliability. Oxford University Press, 2001.

[3] J.-M. Mencik and M. N. Ichchou. Wave finite elements in guided elastodynamics with internal fluid. International Journal of Solids and Structures, 44(7-8):21482167, 2007.

[4] D. J. Mead. A general theory of harmonic wave propagation in linear periodic systems with multiple coupling. Journal of Sound and Vibration, 27(2):235-260, 1973.

[5] D. J. Mead. Wave propagation and natural modes in periodic systems: I. Monocoupled systems. Journal of Sound and Vibration, 40(1):1-18, 1975.

[6] W. X. Zhong and F. W. Williams. On the direct solution of wave propagation for repetitive structures. Journal of Sound and Vibration, 181(3):485-501, 1995.

[7] D. Duhamel, B. R. Mace, and M. J. Brennan. Finite element analysis of the vibrations of waveguides and periodic structures. Journal of Sound and Vibration, 294:205-220, 2006.

[8] L. Houillon, M. N. Ichchou, and L. Jezequel. Wave motion in thin-walled structures. Journal of Sound and Vibration, 281(3-5):483-507, 2001.

[9] B. Mace, D. Duhamel, M. J. Brennan, and L. Hinke. Finite element prediction of wave motion in structural waveguides. Journal of the Acoustical Society of America, 117(5):2835-2843, 2005.

[10] J.-M. Mencik and M. N. Ichchou. A substructuring technique for finite element wave propagation in multi-layered systems. Computer Methods in Applied Mechanics and Engineering, 197(6-8):505-523.

[11] J.-M. Mencik and M. N. Ichchou. Multi-mode propagation and diffusion in structures through finite elements. European Journal of Mechanics - A/Solids, 24(5):877-898, 2005.

[12] L. Brillouin. Wave Propagation in Periodic Structures. Mc Graw Hill Publishing Company, New York, 1946. 